\title{
EARLY CEREBRAL HYPOPERFUSION IN PATIENTS WITH ORTHOSTATIC INTOLERANCE WITHOUT TACHYCARDIA DURING HEAD-UP TILT TEST IS INDEPENDENT OF VASOVAGAL RESPONSE
}

\author{
J. Antonio González-Hermosillo ${ }^{1}$, Alan Rubio-Vega ${ }^{1}$, Karla A. F. González-Olvera ${ }^{1}$, \\ Manuel Sierra-Beltrán ${ }^{1}$, Andrei Kostine ${ }^{1}$, and Claudia Lerma ${ }^{2 *}$ \\ Departments of ${ }^{1}$ Cardiovascular Dysautonomia and ${ }^{2}$ Electromechanical Instrumentation, Instituto Nacional \\ de Cardiología Ignacio Chávez, Mexico City, Mexico
}

\begin{abstract}
Background: Cerebral hypoperfusion before syncope has been shown in patients with chronic orthostatic intolerance (OI) without tachycardia, but it is unknown if an initial decrease of cerebral blood flow velocity (CBFv) could be related to the vasovagal response (VVR) to head-up tilt test (HUTT). Objective: The objective of the study was to compare cardiovascular, cerebrovascular, and autonomic variables during HUTT in Ol patients with or without a VVR. Methods: We included 74 subjects ( $58 \%$ female, mean age $33 \pm 12$ years) who underwent a 30-min HUTT and were divided into three groups: Ol with VVR positive (VVR+), Ol without VVR negative (VVR-), and asymptomatic healthy subjects with negative HUTT (control group). Cardiovascular, cerebrovascular, and autonomic variables were assessed beat-to-beat during HUTT with a Task Force monitor and a trans-cranial Doppler. Mean values were evaluated at baseline and throughout the first 10 min of tilting. Results: Cardiovascular variables were similar in the three groups. Systolic, diastolic, and mean CBFv were similar in VVR+ and VVR-, but both groups had lower CBFv than the control group. Systolic and diastolic CBFv decreased from baseline since min 1 in VVR+ and VVR- and since min 5 in the control group. The mean CBFv had a significant decrease since min 1 compared to baseline in all groups. Spectral indices of heart rate and blood pressure variability showed a similar autonomic response to HUTT in all groups. Conclusion: Patients with chronic Ol without tachycardia have early postural cerebral hypoperfusion, regardless of the VVR during HUTT. (REV INVEST CLIN. 2021;73(6):388-98)
\end{abstract}

Key words: Orthostatic intolerance. Reflex syncope. Cerebral hypoperfusion. Head-up tilt test.

*Corresponding author:

Claudia Lerma

E-mail: dr.claudialerma@gmail.com
Received for publication: 12-04-2021

Approved for publication: 20-05-2021

DOI: $10.24875 /$ RIC.21000199

0034-8376 / (c) 2021 Revista de Investigación Clínica. Published by Permanyer. This is an open access article under the CC BY-NC-ND license (http://creativecommons.org/licenses/by-nc-nd/4.0/). 


\section{INTRODUCTION}

Chronic orthostatic intolerance (OI), a heterogeneous entity associated with the alteration of adaptive mechanisms during the orthostatic challenge, is manifested by the instability of blood pressure (BP) and heart rate (HR) when adopting the upright position. $\mathrm{Ol}$ is characterized by symptoms such as dizziness, weakness, nausea, headache, palpitations, sweating, presyncope, or syncope, which are improved or resolved by lying down ${ }^{1,2}$. In most patients with $\mathrm{Ol}$, the disorder is mild and improves over time, but some of them have more severe symptoms that could be disabling 3,4 .

Ol involves a sympathetic dysfunction because the autonomic nervous system regulation fails to maintain an adequate increase in HR, cardiac contractility, and peripheral vasoconstriction. Therefore, during upright posture, the gravitational pooling of blood in the lower body induces an excessive BP fall with a decrease of cardiac output (CO) and cerebral perfusion ${ }^{3-5}$.

Head-up tilt test (HUTT) permits to identify different types of Ol according to hemodynamic cardiovascular, cerebrovascular, and autonomic responses ${ }^{1}$. According to an international consensus statement ${ }^{6}$, postural tachycardia syndrome (POTS) is characterized by a sustained HR increment $\geq 30$ beats/min within 10 min of standing or head-up tilt in the absence of orthostatic hypotension. The standing HR for all subjects is often $\geq 120$ beats $/ \mathrm{min}$. This condition is different from the classical reflex syncope in which there is a relatively sudden change in autonomic nervous system activity leading to a fall in BP, HR, and cerebral perfusion. Chronic Ol without tachycardia is a distinct entity that shares many features with POTS without the excess upright tachycardia, with milder dysautonomia ${ }^{7}$.

Orthostatic cerebral hypoperfusion syndrome (OCHOs) was defined as symptomatic reduction in the cerebral blood flow velocity (CBFv) during the first $10 \mathrm{~min}$ of HUTT, without significant changes in HR, BP, and respiratory pattern ${ }^{8}$. Cerebral hypoperfusion has been observed during HUTT in adult patients with OI leading to reflex syncope ${ }^{9}$.

Syncope is the transient loss of consciousness and postural tone secondary to cardiovascular collapse and generalized cerebral hypoperfusion due to sympathetic efferent activity withdrawal1,10,11. Although the autonomic nervous system dysregulation precedes syncope in patients with Ol, fainting is infrequently observed during HUTT $3,12,13$.

Continuous BP recordings during an orthostatic challenge allow dividing the sequence of hemodynamic events leading to vasovagal response (VVR) (presyncope/syncope) into four phases ${ }^{14}$. Phase 1 , early stabilization of $\mathrm{CO}$ and mean blood pressure (mBP), is almost complete within 2-3 min after tilting, due to an increase of $\mathrm{HR}$ and/or systemic vascular resistance (SVR) resulting from increased efferent sympathetic activity. Phase 2 is characterized by circulatory instability with increased BP variability with or without a progressive fall in SVR, CO, or both. After a variable time-interval, Phase 3 , manifested by terminal hypotension and syncope, may occur. This phase is not necessarily observed in all patients with Ol. Cerebral hypoperfusion (e.g., dizziness, confusion, blurred vision, and presyncope/syncope) always occurs when the cerebral autoregulation fails to compensate for the BP fall ${ }^{9,14}$. However, during Phase 2 , early cerebral hypoperfusion has been observed in some patients with $\mathrm{Ol}$, independently of $\mathrm{BP}, \mathrm{HR}$, and respiratory changes ${ }^{8}$.

To the best of our knowledge, it has not been determined if the early cerebral hypoperfusion observed in some patients with chronic Ol without tachycardia is related to the final circulatory collapse (VVR) leading to reflex syncope. Therefore, the purpose of this study was to compare cardiovascular, cerebrovascular, and autonomic variables during the first $10 \mathrm{~min}$ of tilting in chronic Ol patients with or without a VVR (presyncope/syncope with hypotension, with or without bradycardia) ${ }^{15}$. We hypothesized that those with a VVR have a greater cerebral hypoperfusion and higher sympathetic activity during Phase 2 compared to those without VVR.

\section{METHODS}

\section{Subjects}

Participants were selected from the database of the Department of Cardiovascular Dysautonomia at the National Institute of Cardiology in Mexico (Instituto 
Figure 1. Flow chart of patients' selection.

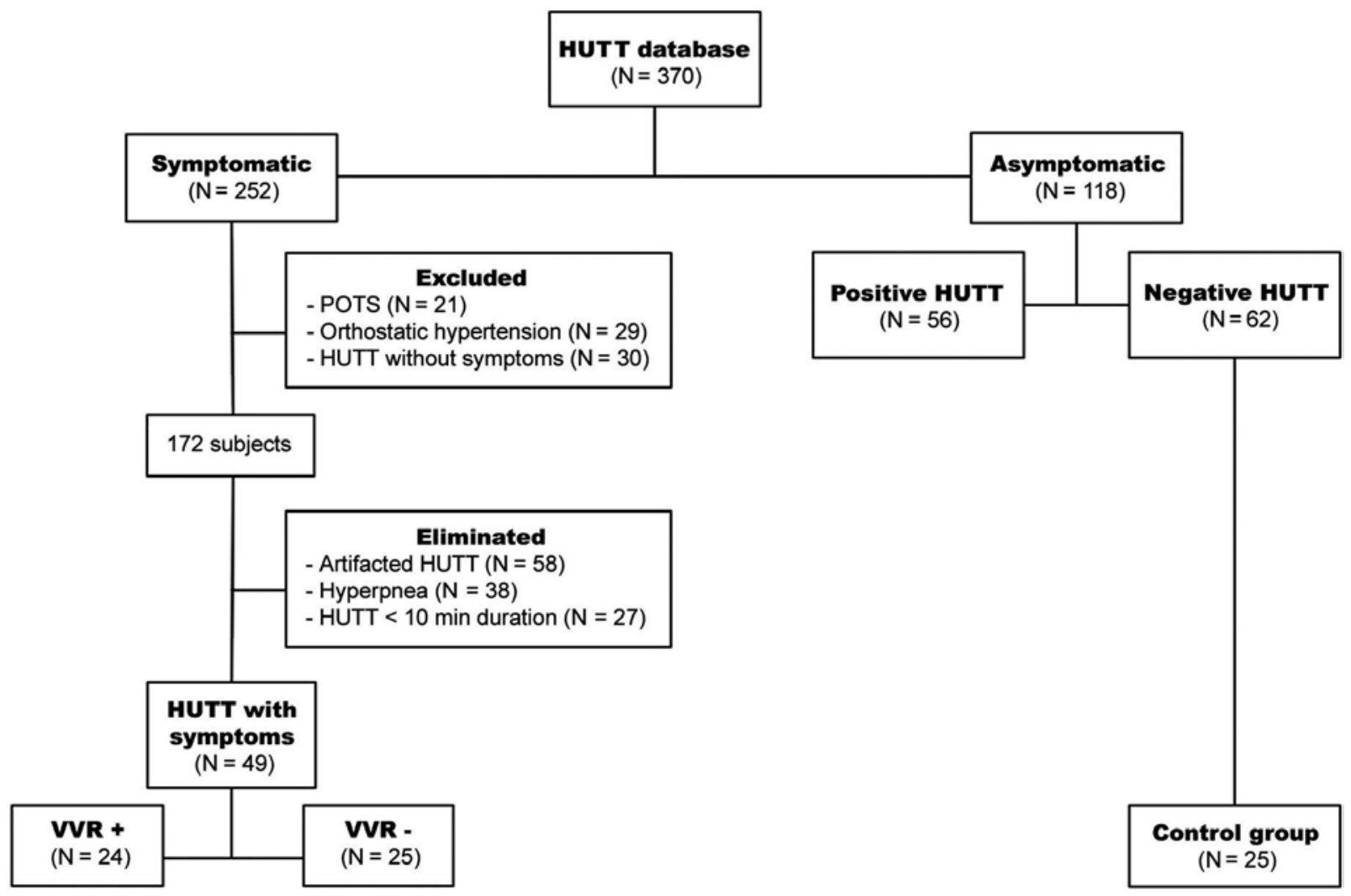

Nacional de Cardiología Ignacio Chávez). The database comprises 370 subjects who underwent a drugfree HUTT. All were screened with a medical history and 12-lead electrocardiogram to exclude those with cardiac, neurologic, or metabolic causes of syncope. We selected 49 patients with suspected Ol and orthostatic symptoms during HUTT, similar to the symptoms they had for at least 1 year before the study (Fig. 1). All of them had a previous history of symptoms during standing (lightheadedness, palpitations, tiredness, weakness, diminished concentration, tremulousness, nausea, visual changes, discomfort in head or neck, throbbing of the head, anxiety, chest discomfort, dyspnea, presyncope, or syncope), relieved by lying down. Patients with POTS, orthostatic hypotension, orthostatic hypertension, and HUTT without symptoms were excluded from the study. Subjects unable to complete at least $10 \mathrm{~min}$ of HUTT for any reason (including syncope), with evidence of hyperventilation (postural hyperpnea), or with artifacts during HUTT were eliminated. Patients were categorized in two groups: (1) with a VVR positive
(VVR+, $\mathrm{n}=24$ ), and (2) without VVR negative (VVR-, $\mathrm{n}=25$ ). The VVR in VVR+ occurred at $19.3 \pm 7.0 \mathrm{~min}$ after tilting, with Type 1 (mixed response, i.e., hypotension with bradycardia) observed in 13 (54\%) patients and Type 3 (vasodepressor response, i.e., hypotension only) in 11 (46\%) patients ${ }^{15}$.

Twenty-five asymptomatic healthy subjects (recruited in a previous study from a sample of volunteer medical students and hospital staff) with negative HUTT $^{16}$ served as a control group. All subjects gave their informed consent for inclusion before participating in the study. The study was conducted in accordance with the Declaration of Helsinki, and the protocol was approved by the Research and Ethics Committee of the National Cardiology Institute Ignacio Chávez (protocol number 18-1052).

\section{HUTT protocol}

All subjects were instructed to discontinue caffeine, alcohol, and tobacco use, and medications with 
cardiovascular/autonomic effects $48 \mathrm{~h}$ before the study, and the daily intakes of water and salt were not restrained. All tilt tests were performed between 8:00 am and 10:00 am after an overnight fast. After 30 min of supine resting, baseline characteristics were assessed during the last $5 \mathrm{~min}$ in the supine position. Thereafter, subjects were tilted to a $70^{\circ}$ upright position. HUTT was interrupted when a VVR was elicited with a fall in systolic BP (sBP) below $80 \mathrm{mmHg}$, or after 30 min of tilting.

BP was recorded continuously using finger arterial plethysmography, and periodically calibrated to brachial artery pressure with an oscillometer in the contralateral arm (Task Force Monitor, TFM: CNSystems, Graz, Austria) ${ }^{17}$. Hemodynamic variables were determined with thoracic bioimpedance cardiography. The variables of interest were obtained on a beat-to-beat basis: mean HR, mBP, sBP, diastolic BP (dBP), stroke index $(\mathrm{SI})$, cardiac index $(\mathrm{Cl})$, and total peripheral resistance index (TPRI). The autonomic modulation was obtained by estimation of the power spectral density of $\mathrm{HR}$ and $\mathrm{dBP}$ variability with an autoregressive method. Artifacts and ectopic activity were eliminated. The absolute powers in the low-frequency band $(0.04-0.15 \mathrm{~Hz})$ and high-frequency band $(0.15-0.4 \mathrm{~Hz})$ were calculated in normalized units ${ }^{18}$. Indexes within the low-frequency band are indicators of sympathetic efferent traffic to the heart (LF), or the peripheral arteries (LFdBP), while the high-frequency index (HF) represents parasympathetic traffic to the heart. The ratio LF/HF, considered as an indicator of sympathovagal, was also obtained.

The CBFv was continuously measured throughout the HUTT with a transcranial Doppler ultrasound (EZDop; DWL, Electronics, Sipplingen, Germany). Through a temporal bone window, the left middle cerebral artery was insonated with a $2 \mathrm{MHz}$ probe. Beat-to-beat measurements were obtained of the following variables: systolic CBFv (sCBFv), diastolic CBFv (dCBFv), mean $\mathrm{CBFv}$ ( $\mathrm{mCBFv}$ ), and cerebrovascular resistance (CVR). The CVR was defined as $\mathrm{mBP} / \mathrm{mCBF}$. The cerebral blood flow category was determined by the $m C B F v$ velocity according to Novak ${ }^{19}$.

All variables were averaged within the following time intervals: last $5 \mathrm{~min}$ of supine resting (baseline), $1^{\text {st }}$ min after tilting ( $\min 1$ ), from min 2 to 6 after tilting (min 5), and from min 6 to 11 after tilting ( $\min 10$ ).

\section{Statistical analysis}

A Kolmogorov-Smirnov test was applied to all continuous variables to verify if they had a normal distribution; only two variables required a logarithmic transformation. Results are presented as mean and standard deviation or as mean and $95 \%$ confidence intervals. Categorical variables are shown as absolute frequency and percentage. Age and body mass index was compared between groups by one-way ANOVA. All other continuous variables were compared with ANOVA for repeated measures to make comparisons between the three groups (controls, VVR+, and VVR-) and within the four periods of time measurements (baseline, 1, 5, and $10 \mathrm{~min}$ of tilt). The $\mathrm{p}$-values of the post hoc analyses were adjusted by the Bonferroni method. $p \leq 0.05$ was considered statistically significant. All the analyses were performed with the program SPSS version 21.0 (IBM Statistics, Armonk N.Y).

\section{RESULTS}

The three studied groups had similar age and body mass index, with fewer women in the control group (Table 1).

\section{Cardiovascular hemodynamics}

Figure 2 shows the mean values of $B P$ and $H R$. At baseline, sBP was lower in the VVR+ group (108.6 \pm $11.8 \mathrm{mmHg}$ ) compared to the control group (117.4 $\pm 11.3 \mathrm{mmHg}$ ), $\mathrm{p}=0.032$. On tilting, $\mathrm{sBP}, \mathrm{dBP}, \mathrm{mBP}$, and $H R$ increased in all groups and remained higher than baseline. At 10 min of tilting, VVR+ patients had lower $\mathrm{sBP}, \mathrm{dBP}$, and $\mathrm{mBP}$ compared to the other two groups.

At baseline, $\mathrm{Cl}, \mathrm{SI}$, and TPRI were similar in all groups (Fig. 3). After tilting, SI decreased, and TPRI increased in all groups, while the $\mathrm{Cl}$ decreased after 5 min of tilt only in the control and VVR-groups.

\section{Cerebrovascular hemodynamics}

Since baseline and throughout the tilting, $\mathrm{SCBFv}$ and $\mathrm{dCBFv}$ were lower in VVR- and VVR+ than in the control group (Fig. 4). As for $m C B F v$ during baseline, it was lower only in VVR+ compared to control. Since min 1 of tilting, mCBFv was lower than control in both 
Table 1. Patient's characteristics and percentage of change of mean cerebral blood flow velocity (mCBFv) evaluated during HUTT. Results are reported as mean \pm standard deviation or absolute value (percentage)

\begin{tabular}{lccc}
\hline Variables & \multicolumn{1}{c}{ Groups } \\
\cline { 2 - 4 } & $\begin{array}{c}\text { Control } \\
(\mathrm{n}=\mathbf{2 5})\end{array}$ & $\begin{array}{c}\text { VVR (-) } \\
(\mathrm{n}=25)\end{array}$ & $\begin{array}{c}\text { VVR (+) } \\
(\mathrm{n}=\mathbf{2 4})\end{array}$ \\
\hline Age (years) & $33 \pm 11$ & $33 \pm 14$ & $31 \pm 12$ \\
Women, $\mathrm{n}(\%)$ & $8(32 \%)$ & $18(72 \%)^{\&}$ & $17(70.8 \%)^{\&}$ \\
Body Mass Index $\left(\mathrm{kg} / \mathrm{m}^{2}\right)$ & $25.5 \pm 3$ & $23.9 \pm 4$ & $24.3 \pm 4$ \\
\hline Percentage of mCBFv change from baseline & $-4.4 \pm 5.2$ & $-11.2 \pm 9.9^{*}$ & $-13.4 \pm 14.7^{*, \&}$ \\
\hline 1 min & $-11.3 \pm 8.7^{*}$ & $-22.0 \pm 17.2^{*, \&}$ & $-21.7 \pm 14.9^{* \& \&}$ \\
5 min & $-16.5 \pm 11.2^{*}$ & $-25.1 \pm 13.5^{*}$ & $-31.2 \pm 19.1^{*, \&}$ \\
10 min & & &
\end{tabular}

${ }^{*} p<0.05$ versus baseline (within same group).

$\& p<0.05$ versus control.

$\operatorname{VVR}(-)$ : Vasovagal response negative.

VVR (+): Vasovagal response positive.

VVR- and VVR+. Conversely, at baseline CVR was similar in groups, and after tilting, it increased in all groups, being higher in VVR- and VVR + compared to the control group.

Table 1 shows the percentage of change in mCBFv during the tilt test as compared to baseline in each group. The mCBFv dropped from baseline in VVR- and VVR + since min 1 of tilting, while in the control groups, the fall started later at min 5 . Compared to controls, VVR+ had a greater drop in mCBFv since the min 1 of tilting, while the decrease in the VVR- group was greater only at the min 5 of tilting. The fall of mCBFv throughout the tilt was similar in VVR- and VVR+ patients.

The cerebral blood flow response to tilt was evaluated according to the OCHOs severity scale. The response in the control group was normal in 12 (48\%) and mild to severe in 13 (53\%) subjects. In the VVR-, the response was normal in only $4(16 \%)$ and mild to severe in 21 ( $84 \%$ ) patients, while the VVR+ had only $2(8 \%)$ patients with normal response and 15 (92\%) patients with mild to severe OCHOs, $(p=0.002)$.

\section{Autonomic variables}

Figure 5 shows that all autonomic variables were similar between groups during the tilt test. LF increased, and HF decreased since min 5 in the VVR+ group, and since min 10 in the three groups. The LF/HF increased versus baseline values since $\min 5$ of tilting in all groups. LFdBP increased compared to baseline values at $\min 10$ in the three groups.

\section{DISCUSSION}

The main findings of the present study are the following: (i) in patients with chronic Ol without tachycardia, there are important changes in the cerebral hemodynamics consisting of significant reduction of CBFv observed since the baseline in supine position, which remained lower than in healthy controls during the first 10 min of HUTT; and (ii) this cerebral hypoperfusion is independent of the VVR observed during the tilt test in some patients with chronic OI. These findings agree with those reported by Novak et al. (2018), who described a distinct form of OI without tachycardia in patients with hypocapnic cerebral hypoperfusion during HUTT20.

Our study included patients with OI without tachycardia, one form of chronic Ol not widely recognized, which has clinical and pathophysiological features similar to POTS 7 . Reduced CBFv with orthostasis precedes hypocapnic hyperpnea and sympathetic activation in POTS patients during orthostatic challenge ${ }^{21}$. It has been shown than in young, normocapnic POTS subjects, CBFv drops by $19.5 \%$ compared with only 
Figure 2. Hemodynamic variables measured during the orthostatic challenge in the studied groups (Control, VVR-: vasovagal response negative; and VVR+: vasovagal response positive). sBP: systolic blood pressure; dBP: diastolic blood pressure; mBP: mean blood pressure; HR: heart rate. These values are reported as mean with $95 \%$ confidence intervals.
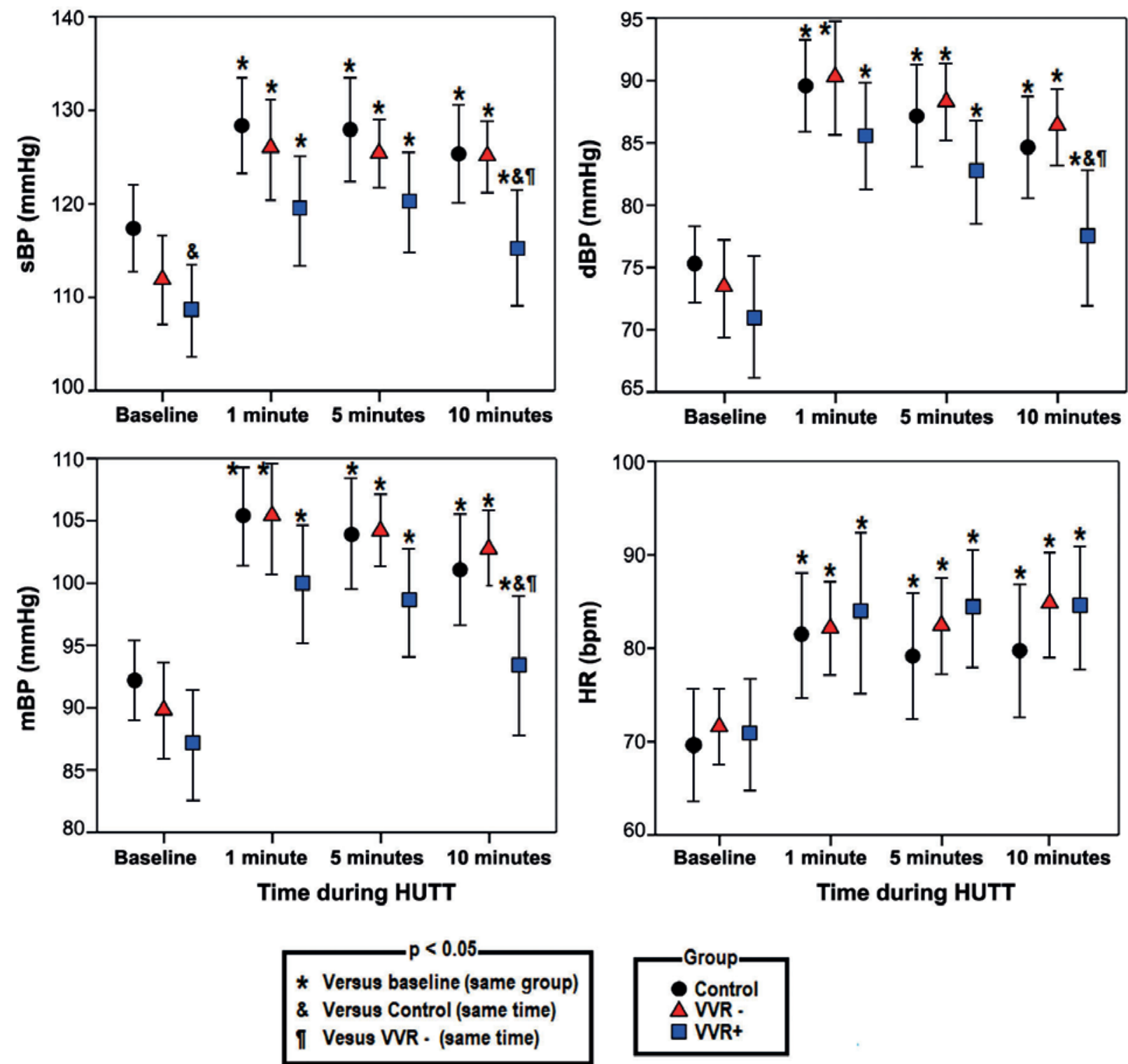

$10.3 \%$ in healthy controls during HUTT. A decrease in the perfusion of the brain may explain the symptoms of lightheadedness, dizziness, and mental confusion that are common in POTS patients. Our group ${ }^{22}$ and others ${ }^{23}$ have observed that increased oscillations in arterial pressure were evident in POTS patients during tilt and appeared to synchronize with increased oscillations in CBFv, which could be related to inefficient autoregulation and increased sympathetic activity. Nevertheless, the sympathetic-mediated cerebral vasoconstriction is controversial: while some studies have found little sympathetic vascular control in the brain ${ }^{24}$, others have found a large sympathetic contribution to cerebral blood flow regulation in humans and mammals ${ }^{25}$.

Bondar et al. stated that a sympathetic-mediated cerebral vasoconstriction could shift the autoregulatory curve to the right ${ }^{26}$. Thus, autoregulation would be efficient at high BP but inefficient at lower BP. If this 
Figure 3. Hemodynamic variables measured during the orthostatic challenge in the studied groups (Control, VVR-: vasovagal response negative; and VVR+: vasovagal response positive). $\mathrm{Cl}$ : cardiac index; SI: stroke index; TPRI: total peripheral resistance index. These values are reported as mean with $95 \%$ confidence intervals.
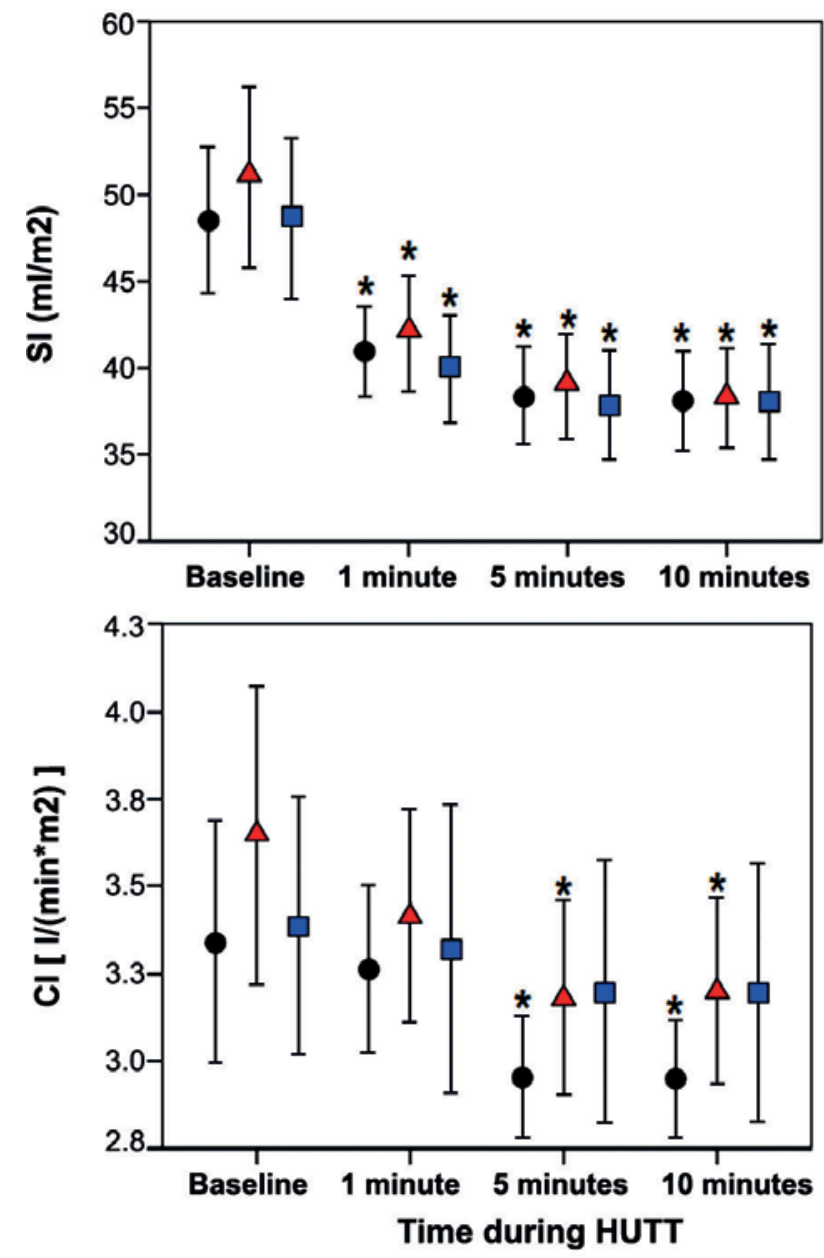

occurred, the decrease in BP seen in POTS subjects during tilt would not be autoregulated, and CBFV would decrease with the decrease in $\mathrm{mBP}$. Ocon et al. ${ }^{23}$ reported that normocapnic patients with POTS have decreased upright cerebral blood flow and cerebral autoregulation during an orthostatic challenge. We believe that the postural reduction in CBFv could be attributed, at least in part, to cerebral vasoconstriction related to an increase in the baroreflex-induced sympathetic outflow to the cerebral circulation. However, in this study, sympathetic nerve function was assessed using only spectral measures of both $\mathrm{HR}$ and BP variability's, which did not show higher sympathetic activity during the orthostatic challenge in subjects with OI without tachycardia as compared to healthy controls. Moreover, we failed to show that

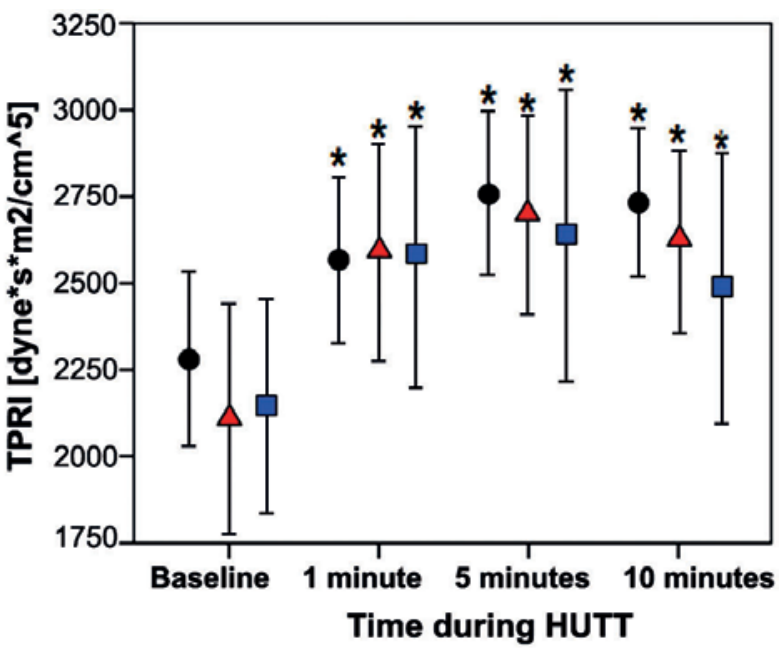

those with a VVR had greater cerebral hypoperfusion and higher sympathetic activity during Phase 2 of HUTT, compared to those without VVR.

The cerebral hypoperfusion in our patients with chronic OI without tachycardia could not be explained by an exaggerated initial central hypovolemia ${ }^{21}$, since they did not show an important fall in SI (i.e., central hypovolemia). Nevertheless, the group of patients with VVR (VVR+) exhibited lower sBP at baseline, and significantly lower sBP, dBP, and $\mathrm{mBP}$ at $10 \mathrm{~min}$ of tilting, which may represent an impaired peripheral vasoconstriction.

$\mathrm{OCHO}$ are a condition associated with orthostatic hypoperfusion. Patients with $\mathrm{OCHOs}$ have stable 
Figure 4. Changes in cerebrovascular variables of each group according to the responses during the orthostatic challenge. (Control, VVR-: vasovagal response negative; and VVR+: vasovagal response positive). sCBFv: systolic cerebral blood flow velocity; dCBFv: diastolic cerebral blood flow velocity; mCBFv: mean cerebral blood flow velocity; CVR: cerebrovascular resistance. These values are reported as mean with $95 \%$ confidence intervals.
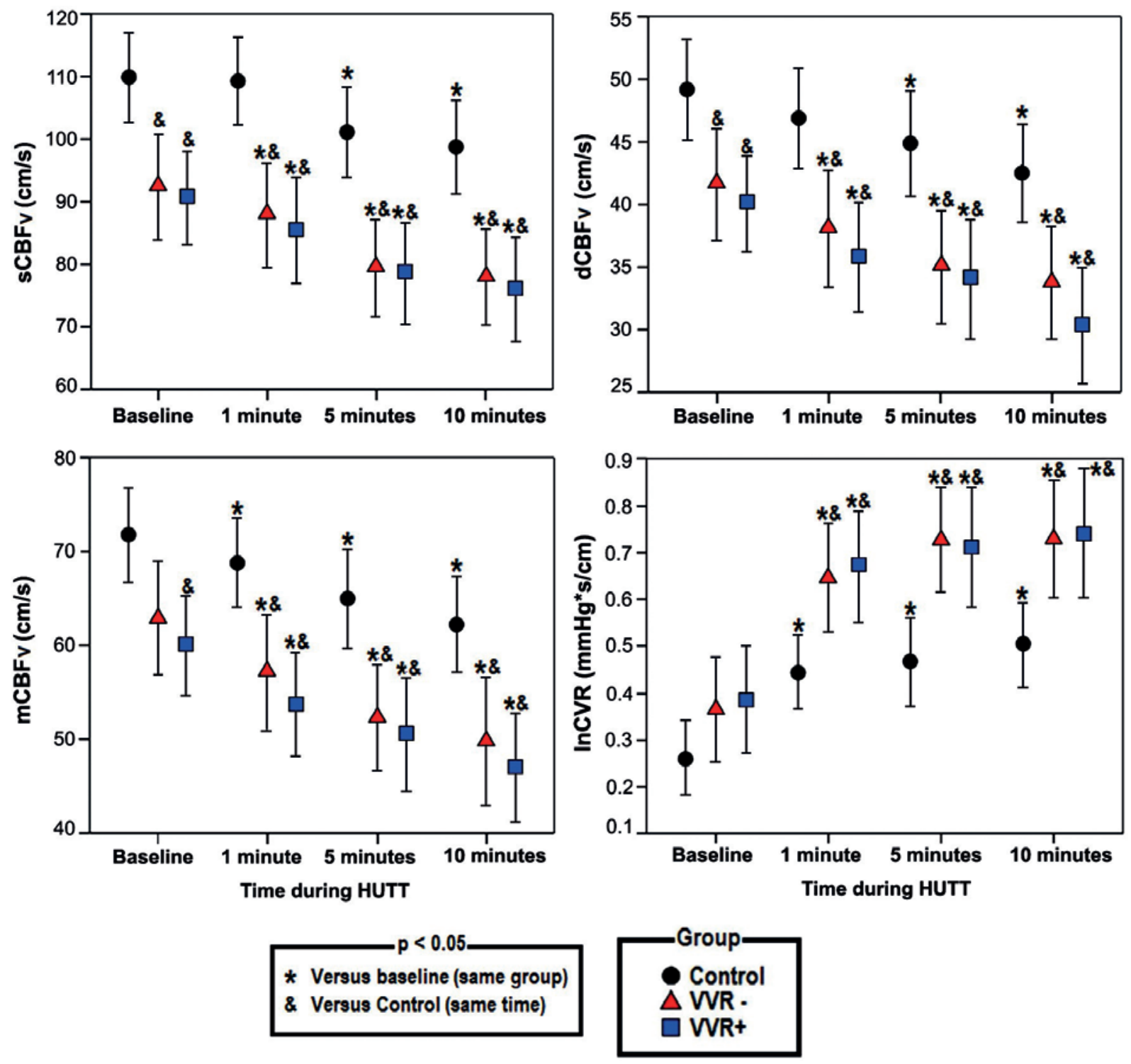

orthostatic BP, HR, and respiratory pattern (normocapnia), and they still have reduced orthostatic $\mathrm{CBFv}^{8}$. The reduction of CBFv was enough to induce symptoms of cerebral hypoperfusion in $25 \%$ of our young patients with Ol without tachycardia, similar to that reported previously by Novak et al. ${ }^{8,27}$ in older patients with $\mathrm{OCHO}$ without orthostatic hypotension (20\%), and by Ocon et al. in POTS (19.5\%) ${ }^{23}$. Our study suggests that $\mathrm{Ol}$ without tachycardia is also associated with cerebral hypoperfusion and vasoconstriction. POTS and $\mathrm{OI}$ without tachycardia have similar OI symptoms, comparable orthostatic decline in CBFv, and are of similar age and gender ${ }^{20}$.

An autoimmune basis for POTS has been described ${ }^{28}$. These observations have led investigators to seek for autoantibodies that might play a role in the pathogenesis of OI. Vernino et al. found ganglionic-receptorbinding antibodies in $7 \%$ of patients with POTS and none in healthy controls ${ }^{29}$. Adrenergic antibodies $(\alpha 1$, 
Figure 5. Changes in autonomic variables of each group according to the responses during the orthostatic challenge. (Control, VVR-: vasovagal response negative; and VVR+: vasovagal response positive). LF: low-frequency of heart rate fluctuations in normalized units; LFdBP: low-frequency of diastolic blood pressure fluctuations in normalized units; HF: high-frequency of heart rate fluctuations in normalized units. These values are reported as mean with $95 \%$ confidence intervals.
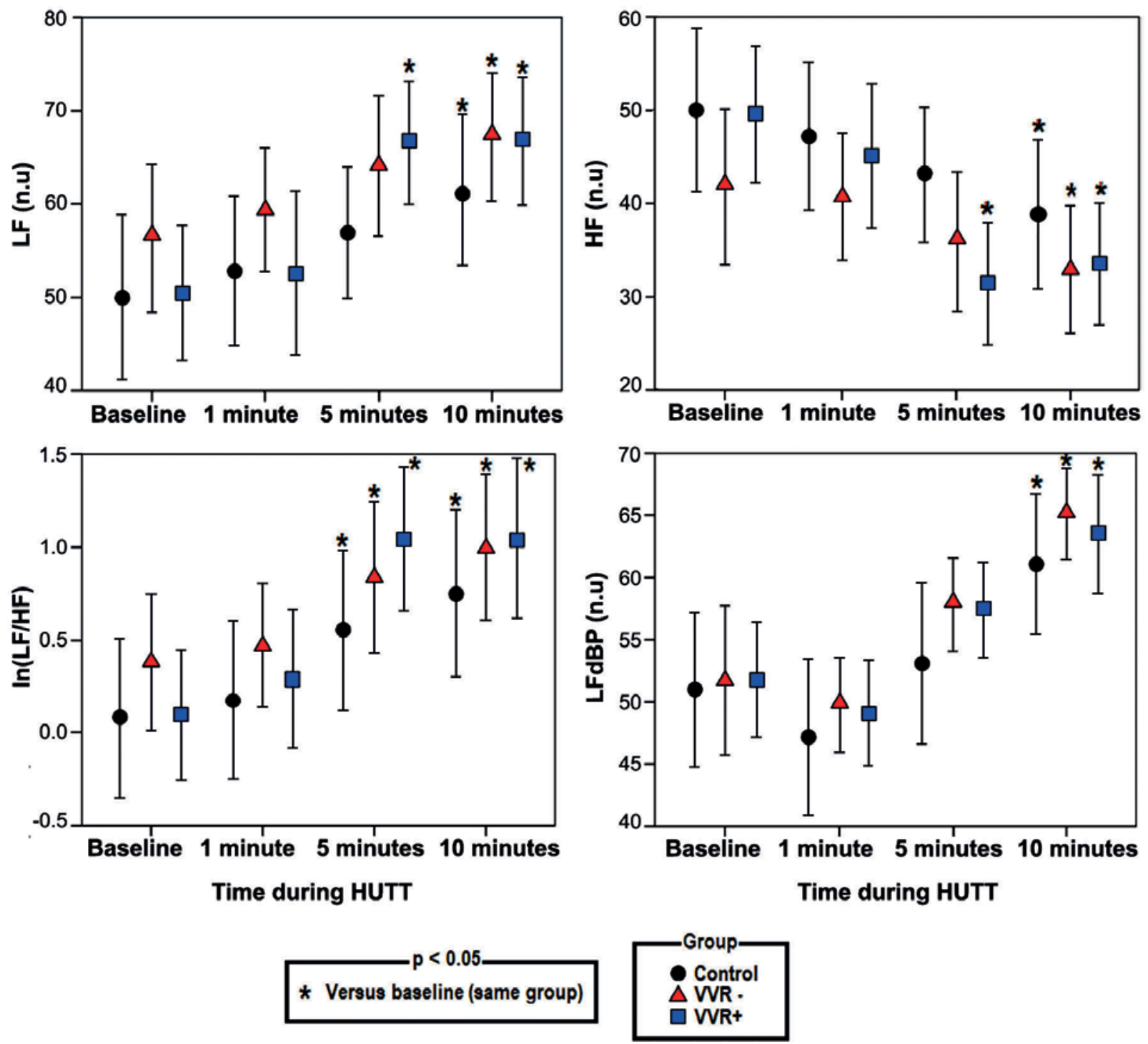

$\beta 1$, and $\beta 2$ ) have been also found in patients with OI syndromes which would lead to a hyperadrenergic state and play a role in these disorders pathophysiology ${ }^{30}$. Fedorowski et al. ${ }^{31}$ demonstrated that serum from subjects with POTS contains autoantibodies, some with a direct stimulatory effect on adrenergic receptors. A majority also exerted an allosterically mediated positive modulatory effect upon $\beta 1 \mathrm{AR}$ and a negative modulatory effect on $\alpha 1$ AR activity, which may explain different constellations of symptoms in POTS.
The study has some limitations; it was retrospective where the diagnostic criteria were applied to already acquired data. Therefore, gender distribution in the control group was different than in the vasovagal positive and negative groups, as there were only $32 \%$ women compared to $67 \%$ and $61 \%$ in both Ol groups. It is well known that orthostatic tolerance is greater in men than in women ${ }^{32}$, which makes difficult to recruit young asymptomatic healthy women for the control group. Further studies are required to show 
whether early cerebral hypoperfusion during orthostasis is more prevalent in women.

Hypocapnia is well known to affect CBFv. In this study, we attempted to control for this factor by only using Ol patients and control subjects who did not exhibit altered respiratory patterns during tilt. Moreover, elucidating the involvement of hypocapnia in the cerebral blood flow autoregulation of OI patients was beyond the scope of the present work. Although the assessment of the autonomic regulation was based on spectral indexes of HR and BP variability's according to the international recommendations ${ }^{18}$, such indirect method may be less sensitive than direct methods (such as microneurography) to elucidate the mechanisms of autonomic regulation in response to tilt ${ }^{33}$. The cerebral blood flow was assessed indirectly using CBFv. Transcranial Doppler measures CBFv instead of blood flow $^{34}$.

Ol represents an important health problem and remains poorly understood. The main significance of these results is the insight that they provide into the events occurring during orthostatic stress, particularly on the changes in CBFv in the period leading up to presyncope/syncope.

The symptoms in patients with chronic Ol without tachycardia may be explained by early postural cerebral hypoperfusion without orthostatic hypotension. Its mechanism is unknown but seems to be due to sympathetic activation alone or combined with hypocapnia. OCHOs are independent of the VVR manifested in some patients by syncope/presyncope. The time lag between the onset of the VVR and the premonitory symptoms excludes the hypothesis that the VVR is dependent on abnormal cerebral hemodynamics.

In conclusion, chronic OI mechanisms are poorly understood. Many patients with OI without tachycardia remain undiagnosed by the tilt test if the CBFv are not measured. Patients with chronic OI without tachycardia have early postural cerebral hypoperfusion. This early postural cerebral hypoperfusion is not related to the final hypotensive response. Then, the question arises whether POTS, OI with VVR+ and VVR- represent different diagnostic entities, or they represent a spectrum of the same disorder of Ol. Identification and treatment of these patients are important, with interventions that could improve their cerebral hemodynamics, and the treatment focused on improving symptoms during orthostasis and not only to prevent syncope.

\section{ACKNOWLEDGMENTS}

The authors thank Betty Lou Chinn, R.N., (Instituto Nacional de Cardiología Ignacio Chávez, Mexico City, Mexico) for reviewing the english style of the manuscript.

\section{REFERENCES}

1. Fedorowski A, Melander O. Syndromes of orthostatic intolerance: a hidden danger. J Intern Med. 2013;273:322-35.

2. Lee $\mathrm{H}$, Kim HA. Orthostatic hypertension: an underestimated cause of orthostatic intolerance. Clin Neurophysiol. 2016; 127:2102-7.

3. Lambert E, Lambert GW. Sympathetic dysfunction in vasovagal syncope and the postural orthostatic tachycardia syndrome. Front Physiol. 2014;5:280

4. Singer W, Mauermann ML, Benarroch EE. Evaluation of autonomic disorders. In: Benarroch EE, editor. Autonomic Neurology. New York: Oxford University Press; 2014. p. 49-70.

5. Hermosillo AG, Márquez MF, Jáuregui-Renaud $K$, Cárdenas M. Orthostatic hypotension, 2001. Cardiol Rev. 2001;9:339-47.

6. Freeman R, Wieling W, Axelrod FB, Benditt DG, Benarroch E, Biaggioni $I$, et al. Consensus statement on the definition of orthostatic hypotension, neurally mediated syncope and the postural tachycardia syndrome. Clin Auton Res. 2011;21:69-72.

7. Parsaik AK, Singer W, Allison TG, Sletten DM, Joyner MJ, Benarroch EE, et al. Orthostatic intolerance without postural tachycardia: how much dysautonomia? Clin Auton Res. 2013; 23:181-8.

8. Novak P. Orthostatic cerebral hypoperfusion syndrome. Front Aging Neurosci. 2016;8:22.

9. González-Hermosillo JA, Petersen A, Salas-Herrera C, BrownEscobar C, Kostine A, Sierra-Beltrán M, et al. Similar cerebral blood flow and autonomic responses to upright rilt rest in adult patients with different hemodynamic mechanisms leading to reflex syncope. J Clin Neurophysiol. 2020;37:239-45.

10. Kapoor WN. Syncope. N Engl J Med. 2000;343:1856-62.

11. Lagi A, Cencetti S, Corsoni V, Georgiadis D, Bacalli S. Cerebral vasoconstriction in vasovagal syncope: any link with symptoms? A transcranial Doppler study. Circulation. 2001;104:2694-8.

12. Soteriades ES, Evans JC, Larson MG, Chen MH, Chen L, Benjamin EJ, et al. Incidence and prognosis of syncope. N Engl J Med. 2002:347:878-85

13. Ganzeboom KS, Mairuhu G, Reitsma JB, Linzer M, Wieling W, van Dijk N. Lifetime cumulative incidence of syncope in the general population: a study of 549 Dutch subjects aged 35-60 years. J Cardiovasc Electrophysiol. 2006;17:1172-6.

14. Jardine DL, Wieling W, Brignole M, Lenders JW, Sutton R, Stewart J. The pathophysiology of the vasovagal response. Heart Rhythm. 2018;15:921-9.

15. Brignole M, Menozzi C, Del Rosso A, Costa S, Gaggioli G, Bottoni $\mathrm{N}$, et al. New classification of haemodynamics of vasovagal syncope: beyond the VASIS classification. Analysis of the presyncopal phase of the tilt test without and with nitroglycerin challenge. Vasovagal Syncope International Study. Europace. 2000;2:66-76.

16. González-Hermosillo A, Sierra-Beltrán M, López-Peña U, Kostin A, Hernández-Pacheco G, Lerma C. Cardiovascular and cerebral hemodynamics in asymptomatic healthy subjects with/without abnormal head-up tilt test versus recurrent fainters. J Clin Neurophysiol. 2018;35:77-83. 
17. Fortin J, Bojic W, Habenbacher W, Grüllenberger R, Heller A, Pacher $\mathrm{R}$, et al. Validation and verification of the Task Force Monitor. Results Clin Stud FDA. 2001;510:1-7.

18. Malik M, Bigger JT, Camm JA, Kleiger RE, Malliani A, Moss AJ, et al. Heart rate variability. Standards of measurement, physiological interpretation, and clinical use. Task force of the European society of cardiology and the North American society of pacing and electrophysiology. Eur Heart J. 1996;17:354-81.

19. Novak P. Quantitative scale for grading of cardiovascular autonomic reflex rests and small fibers from skin biopsies (QASAT). ] Neurol Disord. 2015:3:1-11.

20. Novak P. Hypocapnic cerebral hypoperfusion: a biomarker of orthostatic intolerance. PLoS One. 2018;13:e0204419.

21. Del Pozzi AT, Schwartz CE, Tewari D, Medow MS, Stewart JM. Reduced cerebral blood flow with orthostasis precedes hypocapnic hyperpnea, sympathetic activation, and postural tachycardia syndrome. Hypertension. 2014;63:1302-8

22. Hermosillo AG, Jordan JL, Vallejo M, Kostine A, Márquez MF, Cárdenas M. Cerebrovascular blood flow during the near syncopal phase of head-up tilt test: a comparative study in different types of neurally mediated syncope. Europace. 2006;8:199-203.

23. Ocon AJ, Medow MS, Taneja I, Clarke D, Stewart JM. Decreased upright cerebral blood flow and cerebral autoregulation in normocapnic postural tachycardia syndrome. Am J Physiol Heart Circ Physiol. 2009;297:H664-73.

24. Strandgaard S, Sigurdsson ST. Counterpoint: sympathetic nerve activity does not influence cerebral blood flow. J Appl Physiol. 2008;105:1366-7.

25. van Lieshout J], Secher NH. Point: counterpoint: sympathetic activity does/does not influence cerebral blood flow. J Appl Physiol (1985). 2008;105:1364-6.
26. Bondar RL, Kassam MS, Stein F, Dunphy PT, Fortney S, Riedesel ML. Simultaneous cerebrovascular and cardiovascular responses during presyncope. Stroke. 1995;26:1794-800.

27. Novak P. Cerebral blood flow, heart rate, and blood pressure patterns during the tilt test in common orthostatic syndromes. Neurosci J. 2016;2016:1-20.

28. Vernino S, Stiles LE. Autoimmunity in postural orthostatic tachycardia syndrome: current understanding. Auton Neurosci. 2018; 215:78-82

29. Vernino S, Low PA, Fealey RD, Stewart JD, Farrugia G, Lennon VA. Autoantibodies to ganglionic acetylcholine receptors in autoimmune autonomic neuropathies. N Engl J Med. 2000; 343:847-55.

30. Li H, Yu X Liles C, Khan M, Vanderlinde-Wood M, Galloway A et al. Autoimmune basis for postural tachycardia syndrome. ] Am Heart Assoc. 2014;3:e000755.

31. Fedorowski A, Li H, Yu X, Koelsch KA, Harris VM, Liles C, et al. Antiadrenergic autoimmunity in postural tachycardia syndrome. Europace. 2017;19:1211-9.

32. Reulecke S, Charleston-Villalobos S, Voss A, Gonzalez-Camarena R, Gaitan-Gonzalez M, Gonzalez-Hermosillo ], et al Cardiovascular variability in young male and female subjects in health and orthostatic intolerance. In: 2018 40th Annual International Conference of the IEEE Engineering in Medicine and Biology Society (EMBC). United States: IEEE; 2018. p. 2957-60.

33. Mano T, Iwase S, Toma S. Microneurography as a tool in clinical neurophysiology to investigate peripheral neural traffic in humans. Clin Neurophysiol. 2006;117:2357-84.

34. Serrador JM, Picot PA, Rutt BK, Shoemaker JK, Bondar RL. MRI measures of middle cerebral artery diameter in conscious humans during simulated orthostasis. Stroke. 2000;31:1672-8. 